\title{
PPAR- $\alpha$ Agonist Fenofibrate Upregulates Tetrahydrobiopterin Level through Increasing the Expression of Guanosine 5' -Triphosphate Cyclohydrolase-I in Human Umbilical Vein Endothelial Cells
}

\author{
Jinbo Liu, ${ }^{1}$ Changlin Lu, ${ }^{2}$ Fuwang Li, ${ }^{3}$ Haining Wang, ${ }^{1}$ Liyun He, ${ }^{4}$ Yanting Hao, ${ }^{4}$ \\ Alex F. Chen, ${ }^{5}$ Huijie An, ${ }^{1}$ Xian Wang, ${ }^{3}$ Tianpei Hong, ${ }^{1}$ and Guang Wang ${ }^{1}$ \\ ${ }^{1}$ Department of Endocrinology, Peking University Third Hospital, Beijing 100191, China \\ ${ }^{2}$ Department of Cardiology, Beijing Tongren Hospital, Beijing 100191, China \\ ${ }^{3}$ Department of Physiology and Pathophysiology, School of Basic Medical Sciences and Key Laboratory of Molecular Cardiovascular \\ Science of Ministry of Education, Peking University Health Science Center, Beijing 100191, China \\ ${ }^{4}$ Department of Cardiovascular Medicine, Peking University Third Hospital, Beijing 100191, China \\ ${ }^{5}$ Department of Surgery, University of Pittsburgh School of Medicine and Vascular Surgery Research, Pittsburgh, PA 15240, USA
}

Correspondence should be addressed to Tianpei Hong, tpho66@bjmu.edu.cn and Guang Wang, drwg6688@yahoo.com.cn

Received 10 June 2011; Revised 4 August 2011; Accepted 4 September 2011

Academic Editor: Nanping Wang

Copyright ( $\odot 2011$ Jinbo Liu et al. This is an open access article distributed under the Creative Commons Attribution License, which permits unrestricted use, distribution, and reproduction in any medium, provided the original work is properly cited.

Tetrahydrobiopterin (BH4) is an essential cofactor for endothelial nitric oxide (NO) synthase. Guanosine $5^{\prime}$-triphosphate cyclohydrolase-I (GTPCH-I) is a key limiting enzyme for BH4 synthesis. In the present in vitro study, we investigated whether peroxisome proliferator-activated receptor $\alpha$ (PPAR- $\alpha$ ) agonist fenofibrate could recouple eNOS by reversing low-expression of intracellular BH4 in endothelial cells and discussed the potential mechanisms. After human umbilical vein endothelial cells (HUVECs) were treated with lipopolysaccharide (LPS) for 24 hours, the levels of cellular eNOS, BH4 and cell supernatant NO were significantly reduced compared to control group. And the fluorescence intensity of intracellular ROS was significantly increased. But pretreated with fenofibrate $(10 \mathrm{umol} / \mathrm{L})$ for 2 hours before cells were induced by LPS, the levels of eNOS, NO, and BH4 were significantly raised compared to LPS treatment alone. ROS production was markedly reduced in fenofibrate group than LPS group. In addition, our results showed that the level of intracellular GTPCH-I detected by western blot was increased in a concentrationdependent manner after being treated with fenofibrate. These results suggested that fenofibrate might help protect endothelial function and against atherosclerosis by increasing level of $\mathrm{BH} 4$ and decreasing production of ROS through upregulating the level of intracellular GTPCH-I.

\section{Introduction}

Nitric oxide (NO) is a gas with a half-life of several seconds. It has a pivotal role in vascular homeostasis, such as regulating vascular tone and blood pressure inhibiting platelet aggregation and proliferation of vascular smooth muscle cells and the expression of inflammatory cytokines. Many studies have established that loss of NO bioavailability is a key feature of endothelial dysfunction preceding the appearance of atherosclerosis $[1,2]$.
Endogenous NO is derived from L-arginine in the catalysis of endothelial NO synthase (NOS), which has binding sites for tetrahydrobiopterin (BH4), L-arginine, heme, flavin adenine dinucleotide (FAD), and flavin mononucleotide (FMN) [3]. BH4 is an essential cofactor for endothelial NOS (eNOS). It not only stabilizes the structure of eNOS but also plays a role in the electron transfer during the production of $\mathrm{NO}[4,5]$. When $\mathrm{BH} 4$ levels are inadequate, eNOS is no longer coupled to L-arginine oxidation, which results in reactive oxygen species (ROS) rather than NO 
production, thereby inducing vascular endothelial dysfunction $[6,7]$. Several studies suggest a possible role for $\mathrm{BH} 4$ in regulating NO-mediated endothelial function. Oral administration of $\mathrm{BH} 4$ can improve endothelial function in hypercholesterolemia patients, diabetic patients, and patients with or without coronary artery disease (CAD) [8-11]. Ozaki et al. showed that chronic overexpression of eNOS does not inhibit, but accelerates, atherosclerosis under hypercholesterolemia and that eNOS dysfunction appears to play important roles in the progression of atherosclerosis in apoE-deficient/eNOS-overexpressing mice (apoE-KO/eNOS-Tg mice). But supplementation with BH4 reduced the atherosclerotic lesion size in apoE-KO/eNOS-Tg mice to the level comparable to apoE-KO mice [12]. Another study showed that oral administration of $\mathrm{BH} 4$ slowed the progression of atherosclerosis in apoE-KO mice [13]. Guanosine $5^{\prime}$-triphosphate cyclohydrolase-I (GTPCH-I), encoded by the GCH-I gene, is the rate-limiting enzyme in $\mathrm{BH} 4$ synthesis. In diabetic GCH-Tg mice, superoxide production from endothelium was markedly reduced compared with that of diabetic mice induced by streptozotocin, and NO-mediated vasodilatation was preserved [14]. Zheng et al. [15] found that gene transfer of GTPCH-I could reverse the $\mathrm{BH} 4$ deficiency and endothelial dysfunction by reducing $\mathrm{O}_{2}{ }^{-}$in low renin mineralocorticoid hypertension. In addition, exposure of bovine aortic endothelial cells to GTPCH-I inhibitors or siRNA markedly reduced BH4 and NO levels but increased superoxide anion levels [16].

Hypolipidemic fibrates are pharmacological compounds which activate peroxisome proliferator-activated receptor $\alpha(\operatorname{PPAR}-\alpha)$, a number of the nuclear hormone receptor superfamily. Many studies showed that PPAR- $\alpha$ agonists have a lot of benefits on endothelial function independent of their hypolipidemic effects. Researchers found that PPAR- $\alpha$ activators play a role in cardiovascular protection through an increase in eNOS expression level and phosphorylation of eNOS ser-1179 and NO production in bovine aortic endothelial cells $[17,18]$. Ryan et al. reported that PPAR$\alpha$ agonist fenofibrate improves endothelial function and reduces arterial stiffness in obese glucose-tolerant men [19]. Recent evidence indicates that recoupling eNOS may be a potential approach augmenting the effects of PPAR- $\alpha$ on eNOS. However, the precise effects of PPAR $\alpha$ agonist on recoupling eNOS and its potential mechanism remain uncertain.

Our previous study demonstrated that homocysteine impairs coronary artery endothelial function by decreasing the level of $\mathrm{BH} 4$ in patients with hyperhomocysteinemia [20]. Our previous study also showed that plasma level of BH4 was significantly increased by PPAR $\alpha$ agonist fenofibrate in patients with hypertriglyceridemia. Moreover, coronary flow velocity reserve (CFVR) was significantly improved with fenofibrate treatment [21].

Despite PPAR- $\alpha$ activation may have favorable endothelium-protecting properties, the precious mechanism on eNOS coupling status remains uncertain. In the present study, we investigated whether PPAR- $\alpha$ agonist fenofibrate could improve the expression of intracellular $\mathrm{BH} 4$ through upregulating GTPCH-I, thus contributing to the recoupling of eNOS.

\section{Materials and Methods}

2.1. Cell Culture. Endothelial cells were isolated from segments of human umbilical cord vein by collagenase digestion. They were cultured in medium 199 supplemented with $10 \%$ fetal calf serum as previously described [22]. The medium was renewed every 2 days until confluence (3-4 days); cells were then detached by incubation in PBS containing $0.05 \%$ trypsin and $0.03 \%$ EDTA for $1 \mathrm{~min}$ at room temperature, washed by centrifugation and reseeded onto 35,60 , or $100 \mathrm{~mm}$ plastic culture dishes for ROS, detection, eNOS, BH4, and GTPCH-I measurement. At early confluence, cells were treated with LPS in the presence of fenofibrate or not as indicated in the figure legends. Only endothelial cells passaged less than six times were used for experiments.

2.2. Measurement of Intracellular BH4. For the measurement of total biopterin, high-performance liquid chromatography (HPLC) was used, as previously described with some modification [23]. Cell lysates were suspended in distilled water containing $1 \mathrm{mM}$ Dithiothreitol, $50 \mathrm{mM}$ Tris- $\mathrm{HCl}(\mathrm{pH}$ 7.4), and $1 \mathrm{mM}$ EDTA, centrifuged at $12000 \mathrm{~g}$ at $4^{\circ} \mathrm{C}$ for $15 \mathrm{~min}$, and then subjected to oxidation in acid and base. The supernatant $(90 \mathrm{ul})$ was transferred to an amber tube, and $10 \mathrm{uL}$ of $1: 1$ mixture of $1.5 \mathrm{M} \mathrm{HClO} 4$ and $2 \mathrm{M} \mathrm{H} 3 \mathrm{PO} 4$ was added, followed by centrifugation at $13000 \mathrm{~g}$ for $10 \mathrm{~min}$ at $4^{\circ} \mathrm{C}$. The supernatant $(90 \mathrm{ul})$ was transferred to a new amber tuber, and $10 \mathrm{uL}$ of iodine solution (1\% iodine and $2 \% \mathrm{KI}$ in $1 \mathrm{M} \mathrm{HCl}$ solution) was added to the process of acid oxidation in order to determine total biopterin (BH4, dihydropterin (BH2), and oxidized biopterin(B)). After mixing and standing for $60 \mathrm{~min}$ in the dark at room temperature, excess iodine was reduced by the addition of $5 \mathrm{uL}$ fresh ascorbic acid $(20 \mathrm{mg} / \mathrm{mL}$ in water $)$. To determine $\mathrm{BH} 2+\mathrm{B}$ by alkaline oxidation, $10 \mathrm{uL}$ of $1 \mathrm{M} \mathrm{NaOH}$ was added to $80 \mathrm{uL}$ extract, and then $10 \mathrm{uL}$ of alkaline iodine solution ( $1 \%$ iodine and $2 \% \mathrm{KI}$ in $1 \mathrm{M} \mathrm{NaOH}$ solution) was added. After mixing and standing for $60 \mathrm{~min}$ in the dark at room temperature, $20 \mathrm{uL}$ of $1 \mathrm{M} \mathrm{H}_{3} \mathrm{PO}_{4}$ was added to acidify alkaline oxidation, and then $5 \mathrm{uL}$ fresh ascorbic acid $(20 \mathrm{mg} / \mathrm{mL}$ in water) was added to reduce excess iodine. Samples oxidized under acidic or alkaline conditions were centrifuged at $13000 \mathrm{~g}$ for $10 \mathrm{~min}$ at $4^{\circ} \mathrm{C}$. The supernatant $90 \mathrm{uL}$ was injected into the column by use of an HPLC system with an autosampler and a fluorescence detector (Agilent 1100). A Hypersil C18 column $(4.6 \mathrm{~mm} \times 250 \mathrm{~mm}, 5 \mathrm{um})$ was used for separation of biopterin with a mobile phase of ration of methanol to water $(5: 95, \mathrm{v} / \mathrm{v})$ running at a flow rate of $1.0 \mathrm{~mL} / \mathrm{min}$. The retention time of biopterin was approximately $7.5 \mathrm{~min}$, and the excitation and emission wave lengths were 350 and $440 \mathrm{~nm}$, respectively. Compounds were quantitated by their peak height in comparison with external standards. And $\mathrm{BH} 4$ concentrations, expressed as $\mathrm{pmol} / \mathrm{mg}$ protein, were calculated by subtracting $\mathrm{BH} 2+\mathrm{B}$ from total biopterin. 
2.3. Measurement of Intracellular eNOS. Level of eNOS was measured by use of ELISA kits according to the manufacturer's protocols (BioPCR, China).

2.4. Measurement of Cell Supernatant NO. NO level was measured by use of an ELISA kit according to the manufacturer's protocols (Jiamay Biotech, China).

2.5. Measurement of Intracelluar ROS Generation. Determination of intracellular oxidant production in endothelial cells was based on the oxidation of an ROS probe dye $2^{\prime}, 7^{\prime}$-dichlorofluorescin diacetate (DCF-DA, $20 \mathrm{umol} / \mathrm{L}$ ) by intracellular ROS, and resulting in the formation of the fluorescent compound 2', $7^{\prime}$-dichlorofluorescin (DCF). And DCF florescence was monitored with a confocal laser scanning microscope (Leica) [24].

2.6. Western Blot Analysis. HUVECs were lysated with celllysis buffer $(150 \mathrm{mM} \mathrm{NaCl}, 100 \mathrm{mM}$ Tris-HCl pH 7.4, $1 \mathrm{mM}$ $\mathrm{Na}_{2}$ EDTA, $1 \%$ Triton-X, $10 \mathrm{ug} / \mathrm{mL}$ aprotinin, $10 \mathrm{ug} / \mathrm{mL}$ pepstatin A, $10 \mathrm{ug} / \mathrm{mL}$ leupeptin, $0.05 \mathrm{M} \mathrm{NaF}, 0.01 \mathrm{M} \mathrm{Na}_{4} \mathrm{O}_{7} \mathrm{P}_{2}$, $1 \mathrm{M} \mathrm{Na}_{3} \mathrm{VO}_{4}$ ) and $1 \mathrm{mM}$ PMSF. The protein content was assayed by BCA protein assay reagent. $40 \mathrm{ug}$ protein were loaded to SDS-PAGE and then transferred to PVDF membranes. After incubation for 1 hour in blocking buffer (5\% skim milk powder in TBS-T), the membranes were incubated with primary antibody (Santa Cruz, USA) with a $1: 1000$ dilution, followed by incubating with fluorescently labeled secondary antibody (Invitrogen, USA). Protein bands were visualized by Odyssey infrared imaging system (LI-COR Biosciences, USA), and the intensity of each bands was measured by the accompanied software. We used control as $100 \%$.

2.7. Statistical Analysis. Dates were reported as means \pm SD. The differences between groups were analyzed by one-way ANOVA, and $P<0.05$ was considered statistically significant.

\section{Results}

3.1. Effects of Fenofibrate on Intracellular eNOS and Cell Supernatant NO. As shown in Figure 1, fenofibrate treatment for 24 hours increased the expression of eNOS protein in a concentration-dependent manner. However, after HUVECs were treated with LPS for 24 hours, the levels of cellular eNOS and cell supernatant NO were significantly reduced by $14.4 \%, 19.9 \%$ (both $P<0.05$ ) compared to control groups, respectively. But pretreated with fenofibrate ( $10 \mathrm{umol} / \mathrm{L}$ ) for 2 hours before cells were induced by LPS, the levels of eNOS and NO were significantly raised compared to LPS treatment (both $P<0.05$ ). We also found that PPAR $\alpha$ blocker GW6471 (10 umol/L) abolished the effect of fenofibrate and PPAR $\alpha$ agonist WY14643 (50 umol/L) could produce a similar effect of fenofibrate on intracellular eNOS and cell supernatant NO. And there was no significant difference between group of WY14643 + LPS and group of fenofibrate + LPS in the levels of eNOS and NO $(P=0.93$, $P=0.12$, resp.).

3.2. Effect of Fenofibrate on Intracellular Level of ROS. As shown in Figure 2, the fluorescence intensity of intracellular

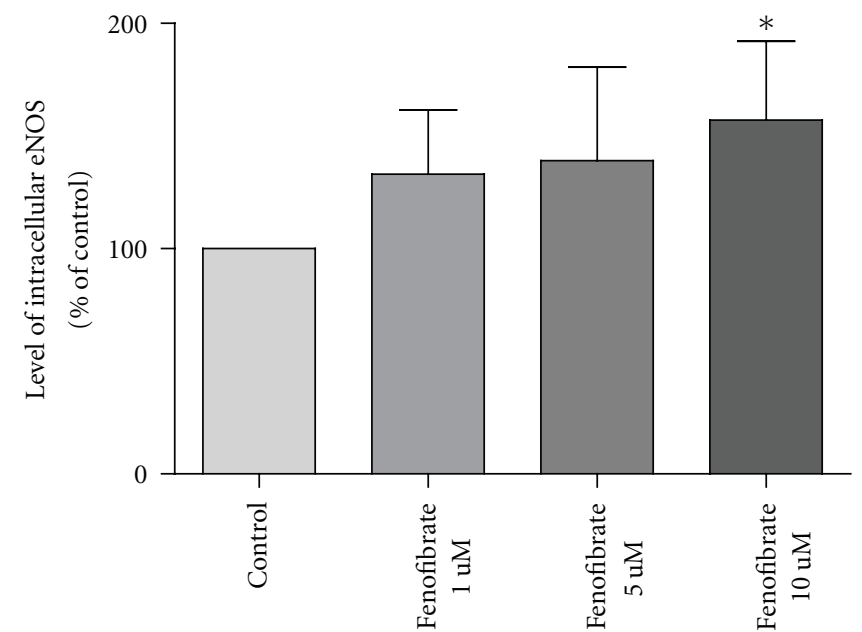

(a)

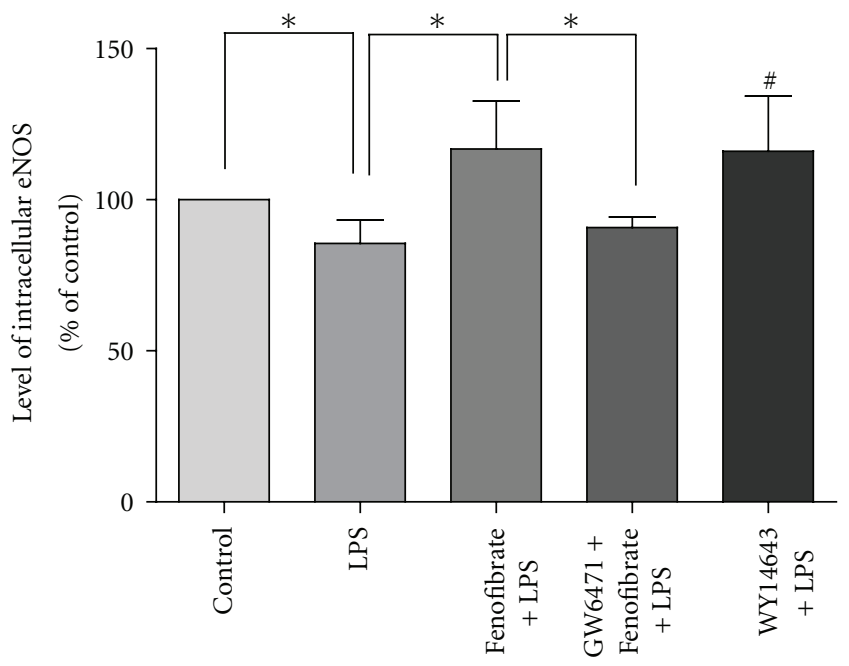

(b)

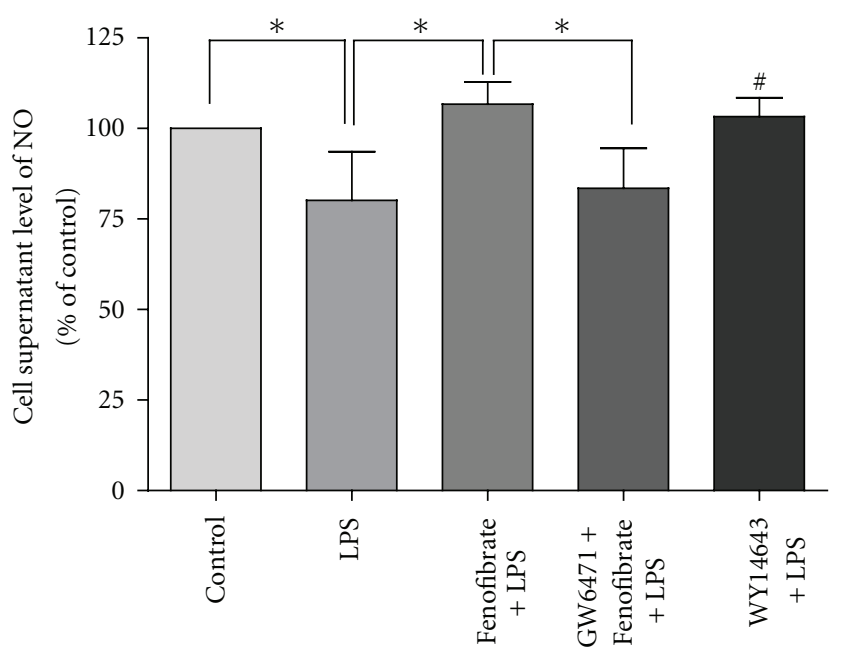

(c)

FIGURE 1: Effect of fenofibrate and related compounds on levels of eNOS (a, b) and NO (c) in control group, LPS group, fenofibrate pretreated group, PPAR $\alpha$ antagonist, and agonist group. eNOS and NO were measured by ELISA kits according to the manufacturer's protocols. Values are expressed as means $\pm \mathrm{SD}$. (a) $* P<0.05$ versus control group. (b, c) ${ }^{*} P<0.05 ;{ }^{*} P<0.05$ versus LPS group. 


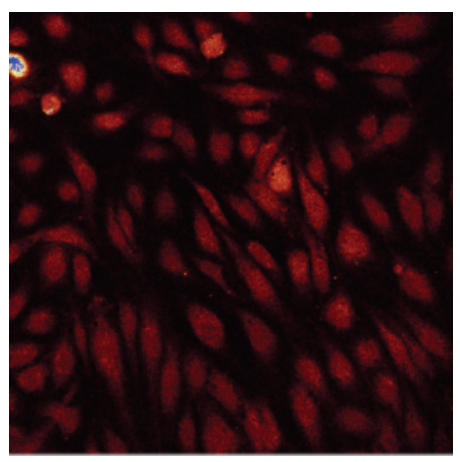

(a)

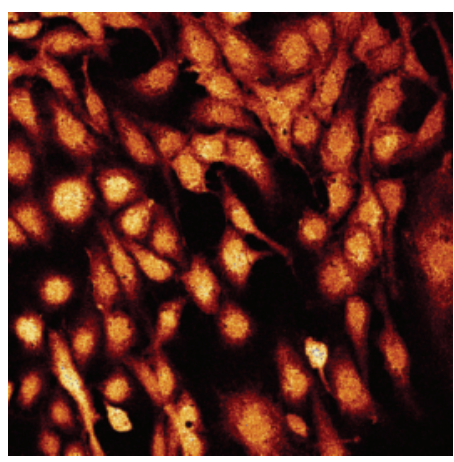

(b)

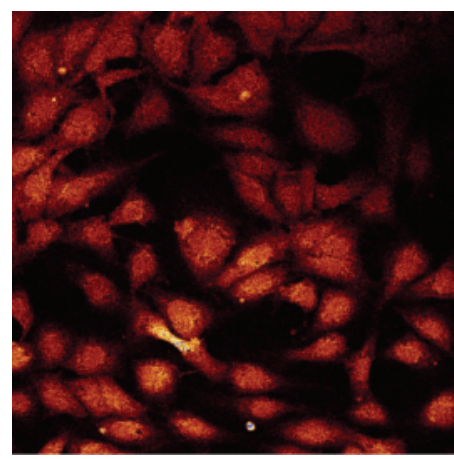

(c)

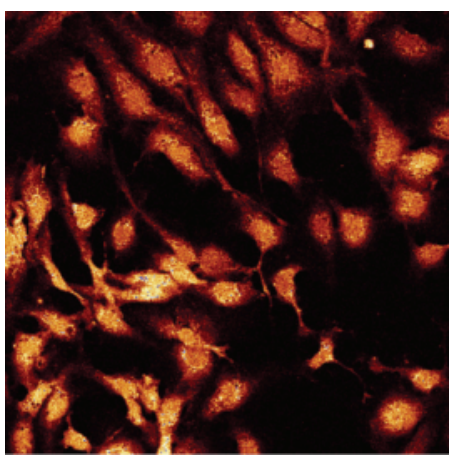

(d)

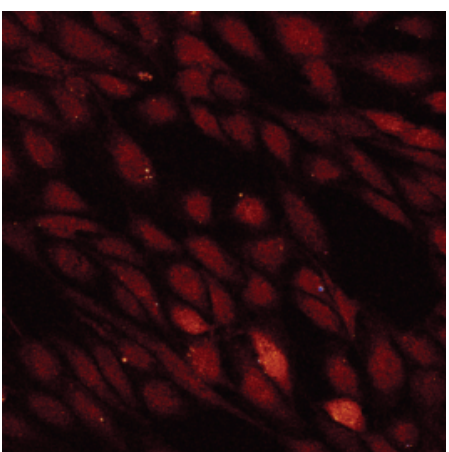

(e)

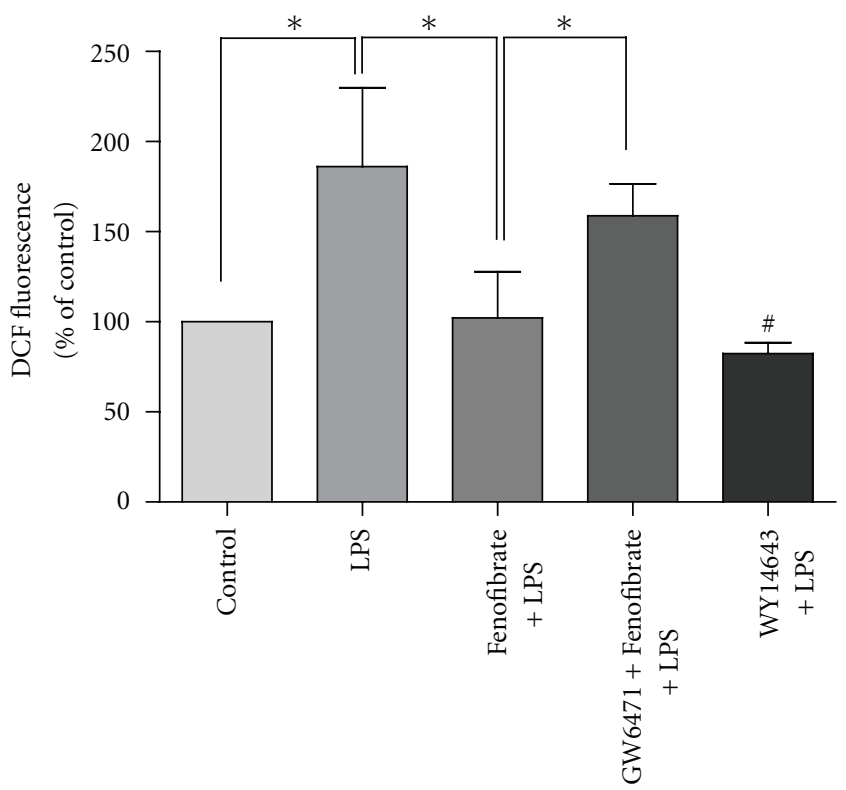

(f)

Figure 2: Effect of fenofibrate and related compounds on ROS formation in cultured endothelial cells. DCFH-DA (20 umol/L) was added to monitor intracellular ROS production with a confocal laser scanning microscope (Leica). (a) Control group; (b) LPS group; (c) fenofibrate + LPS; (d) GW6471 + fenofibrate + LPS; (e) WY14643 + LPS; (f) statistical graph. Values are expressed as means \pm SD; $n=6$. ${ }^{*} P<0.05 ;{ }^{*} P<0.05$ versus LPS group. 
ROS was stronger in LPS group. However, if cells were pretreated with fenofibrate for 2 hours before being incubated with LPS for 24 hours, ROS fluorescence intensity was significantly reduced in fenofibrate group than LPS group. In addition, the fluorescence intensity was stronger than fenofibrate group after being treated by PPAR $\alpha$ antagonist GW6471 (10 umol/L). We also found that PPAR $\alpha$ agonist WY14643 (50 umol/L) could reduce the level of intracellular ROS similarly to the effect of fenofibrate. And there was no significant difference between group of WY14643 + LPS and group of fenofibrate + LPS in the level of ROS $(P=0.25)$.

3.3. Effect of Fenofibrate on Intracellular Level of BH4. $\mathrm{BH} 4$ is an essential cofactor for eNOS. When BH4 levels are inadequate, eNOS is no longer coupled to L-arginine oxidation, which results in ROS rather than NO production. From above statements we knew that LPS stimulation could cause more production of ROS in HUVECs, so we next investigated whether the action of LPS was from the pathway of BH4. As shown in Figure 3, intracellular BH4 level was significantly lower in LPS group than control group $(P<$ 0.05). However, pretreated with fenofibrate $(10 \mathrm{umol} / \mathrm{L})$ for 2 hours, intracellular BH4 level was significantly higher than LPS group $(P<0.05)$. PPAR $\alpha$ blocker GW6471 $(10 \mathrm{umol} / \mathrm{L})$ abolished the effect of fenofibrate on intracellular BH4. And PPAR $\alpha$ agonist WY14643 (50 umol/L) could upregulate the level of intracellular BH4 similarly to fenofibrate without significant difference $(P=0.25)$.

3.4. Effect of Fenofibrate on Intracellular Level of GTPCHI. GTPCH-I is the rate-limiting enzyme in BH4 synthesis. We next investigated whether fenofibrate promoted the expression of BH4 through increasing the level of GTPCH-I in HUVECs. As shown in Figure 4, fenofibrate treatment for 24 hours increased GTPCH-I expression in a concentrationdependent manner. The level of GTPCH-I after fenofibrate treatment reached maximum at 12 hours and maintained at a high level for a long time. We also found that PPAR $\alpha$ blocker GW6471 (10 umol/L) abolished the effect of fenofibrate and PPAR $\alpha$ agonist WY14643 (50 umol/L) could produce a similar effect of fenofibrate on intracellular GTPCH-I. And there was no significant difference between WY14643 group and fenofibrate group in the expression of GTPCH-I $(P=$ $0.81)$.

\section{Discussion}

BH4 is an essential cofactor for eNOS which catalyzes Larginine to L-citrulline with the production of $\mathrm{NO}$, one of the most important vasodilators. Our study demonstrated that endothelial cellular eNOS and NO were significantly reduced when incubated with LPS, accompanied by more ROS production and lower level of $\mathrm{BH} 4$. However, when pretreated with fenofibrate before exposing to LPS, cellular levels of eNOS, NO, and BH4 were all significantly higher than LPS group. There was lower level of ROS in the fenofibrate group than LPS group. Fenofibrate treatment for 24 hours increased GTPCH-I expression in a concentration-dependent manner. Finally, PPAR $\alpha$ antagonist GW6471 could abolish the effects of fenofibrate on these indicators, and PPAR $\alpha$ agonist

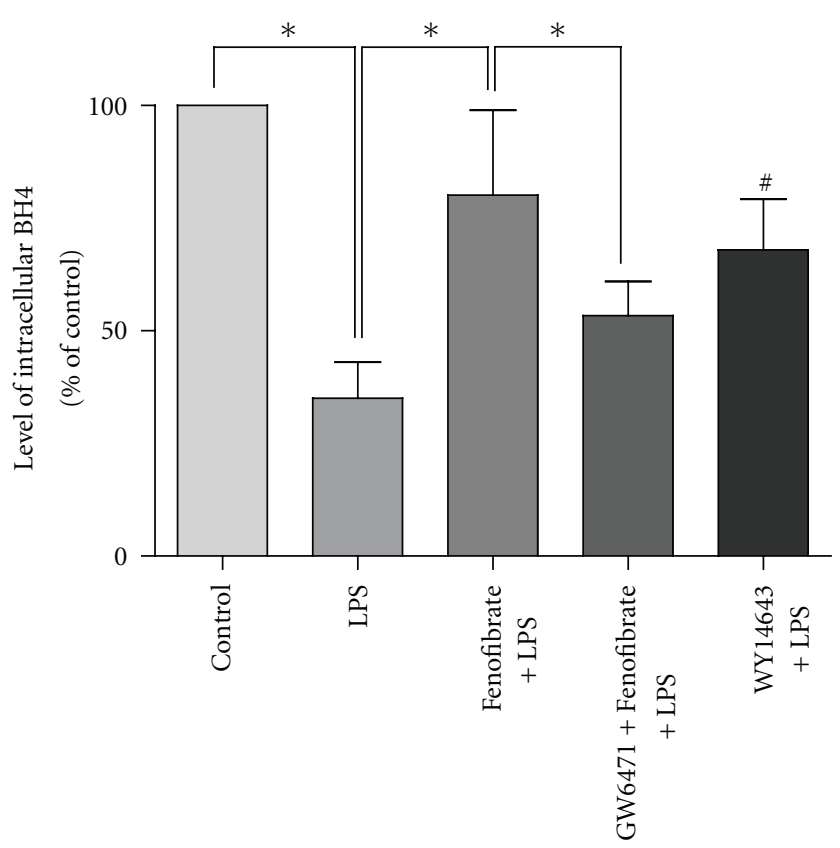

FIGURE 3: Effect of fenofibrate and related compounds on intracellular level of BH4 in each group, measured by HPLC. Values are expressed as means \pm SD. BH4: tetrahydrobiopterin. $n=5$. ${ }^{*} P<0.05$; $P<0.05$ versus LPS group.

WY14643 could produce the similar effect of fenofibrate. Thus, fenofibrate may help protect endothelial function by promoting level of $\mathrm{BH} 4$ and decreasing production of ROS through increasing the level of intracellular GTPCH-I.

Endothelial-derived NO is essential in the maintenance of vascular homeostasis. It has many biological effects, such as suppressing platelet aggregation, leukocyte migration and celluar adhesion to the endothelium, and inhibiting inflammatory response and vascular smooth muscle cell proliferation. Hence, $\mathrm{NO}$ is an anti-atherosclerotic molecule. So loss of NO bioavailability is a key feature of endothelial feature of endothelial dysfunction preceding the appearance of atherosclerosis $[1,25]$. Hypolipidemic fibrates are pharmacological compounds which activate PPAR- $\alpha$, a number of the nuclear hormone receptor superfamily. Many studies showed that PPAR- $\alpha$ agonists have a lot of benefits on vascular endothelial function independent of their hypolipidemic effects. Researchers found that PPAR- $\alpha$ activators play a role in cardiovascular protection through an increase in eNOS expression level and phosphorylation of eNOS ser-1179 and NO production in Bovine aortic endothelial cells $[17,18]$. In our present study, we showed that fenofibrate improved the levels of eNOS and NO in endothelial cells. Tetrahydrobiopterin (BH4) is an essential cofactor that maintains the normal function of eNOS. When BH4 level is inadequate, it could transform eNOS into an oxidant production of ROS. BH4 may improve NOmediated endothelial function, thereby potentially reducing the development of atherosclerosis. Several studies showed that oral administration of $\mathrm{BH} 4$ can improve endothelial function in hypercholesterolemia patients, diabetic patients, 


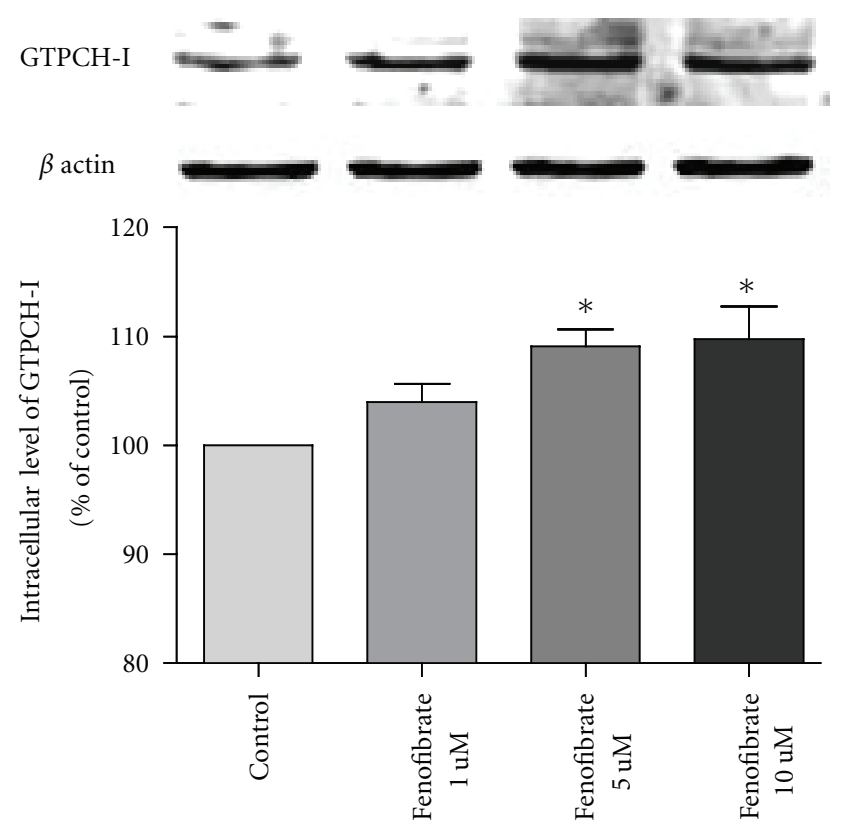

(a)

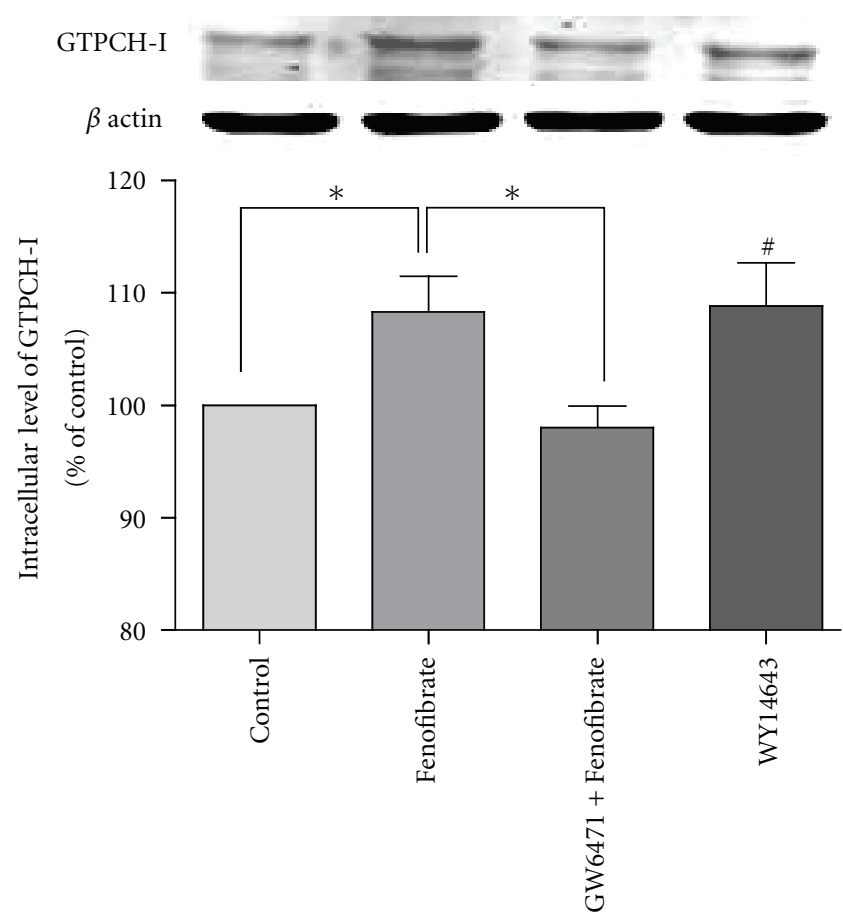

(c)

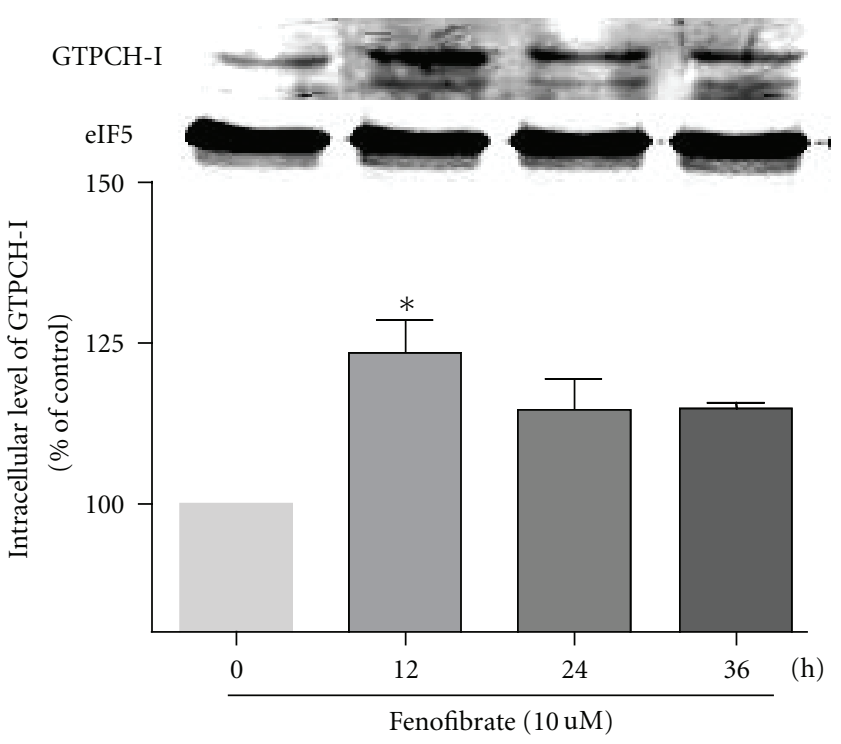

(b)

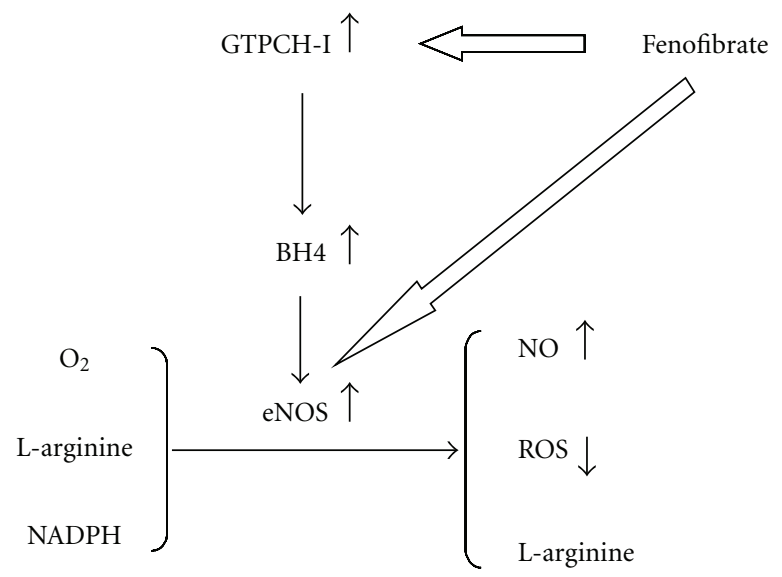

Figure 4: Fenofibrate increases GTPCH-I protein levels in HUVECs, as demonstrated by Western blot analyses. (a) Cells were treated for $24 \mathrm{~h}$ with increasing concentrations of fenofibrate. (b) The level of GTPCH-I after fenofibrate treatment reached maximum at 12 hours and maintained a high level for a long time. (c) GW6471 antagonizes fenofibrate-induced increase in GTPCH-I protein level, WY14643 increases GTPCH-I protein level similarly to the effect of fenofibrate. Dates are means \pm SD in three experiments. (a,b): ${ }^{*} P<0.05$ versus control group; (c) ${ }^{*} P<0.05,{ }^{\#} P<0.05$ versus control group. (d) Proposed scheme: Fenofibrate not only promotes the expression of eNOS, but also up-regulates the level of GTPCH-I, contributing to the increase of BH4, which could promote the activity of eNOS, thus resulting the increase of NO and decrease of ROS. 
and patients with or without coronary artery disease (CAD) [8-11]. Then, correction of endothelial dysfunction may explain in part the beneficial effect of PPAR- $\alpha$ agonist treatment. Our previous study demonstrated that PPAR- $\alpha$ agonist fenofibrate increased the plasma levels of $\mathrm{BH} 4$ and NO in patients with hypertriglyceridemia [21]. Moreover, homocysteine impairs coronary artery endothelial function by inhibiting tetrahydrobiopterin in patients with hyperhomocysteinemia [20]. We hypothesized that fenofibrate could promote the activity of eNOS through restoring the level of $\mathrm{BH} 4$ contributing to lower level of ROS. As mentioned earlier, when treated with fenofibrate, the intracellular level of BH4 was significantly improved compared to LPS treatment alone, accompanied by lower ROS generation. These results suggest that fenofibrate might have potentially beneficial effects in endothelial function by promoting the level of BH4.

Next, GTPCH-I is the rate-limiting enzyme in BH4 synthesis. In many gene transfer animal models of GTPCH-I, such as diabetic GCH-Tg mice and DOCA-salt hypertension rat, overexpression of GTPCH-I could reverse the BH4 deficiency and endothelial dysfunction by reducing superoxide anion production and NO-mediated vasodilatation was preserved [14, 15]. Wang et al. [16] found that exposure of bovine aortic endothelial cells to GTPCH-I inhibitors or siRNA markedly reduced $\mathrm{BH} 4$ and $\mathrm{NO}$ levels but increased superoxide levels. Some drugs such as statin and metformin can improve endothelial function through increasing level of $\mathrm{BH} 4$ by different mechanisms, such as increasing the level of GTP cyclohydrolase I (GTPCH I), suppressing $26 \mathrm{~s}$ proteasome-mediated GTPCH I degradation to increase the level of BH4, and so on [26-28]. In our system, we discovered that fenofibrate treatment increased GTPCH-I expression in a concentration-dependent manner. Then, fenofibrate might play its endothelial protecting and antiatherosclerosis effect by up-regulating BH4 and GTPCH-I levels in HUVECs, which could provide a new perspective for the therapy of endothelial dysfunction (Figure 4(d)). However, the precise mechanism of this process needs to be further investigated.

In conclusion, fenofibrate contributes beneficial effect to prevent endothelial dysfunction from upregulating level of $\mathrm{BH} 4$ and decreasing production of ROS through the mechanism of increasing the level of intracellular GTPCH-I. Fenofibrate may help protect against atherosclerosis potential by promoting the re-coupling of eNOS with normalizing endothelial disorders.

\section{Conflict of interests}

No conflict of interests, financial or otherwise, is declared by the authors.

\section{Acknowledgment}

This work was supported by grants from the Major National Basic Research Program of China (no. 2011CB503904), the Chinese National Natural Science Foundation (no. 30730042) to X. Wang, and the Chinese National Natural Science Foundation (no. 30770873, no. 81070244) to G.
Wang. Jinbo Liu and Changlin Lu contributed equally to this paper.

\section{References}

[1] C. Napoli, F. de Nigris, S. Williams-Ignarro, O. Pignalosa, V. Sica, and L. J. Ignarro, "Nitric oxide and atherosclerosis: an update," Nitric Oxide, vol. 15, no. 4, pp. 265-279, 2006.

[2] M. Félétou and P. M. Vanhoutte, "Endothelial dysfunction: a multifaceted disorder," American Journal of Physiology, vol. 291, no. 3, pp. H985-H1002, 2006.

[3] F. Cosentino and T. F. Lüscher, "Tetrahydrobiopterin and endothelial nitric oxide synthase activity," Cardiovascular Research, vol. 43, no. 2, pp. 274-278, 1999.

[4] B. R. Crane, A. S. Arvai, D. K. Ghosh et al., "Structure of nitric oxide synthase oxygenase dimer with pterin and substrate," Science, vol. 279, no. 5359, pp. 2121-2126, 1998.

[5] D. J. Stuehr, C. C. Wei, Z. Wang, and R. Hille, "Exploring the redox reactions between heme and tetrahydrobiopterin in the nitric oxide synthases," Dalton Transactions, no. 21, pp. 34273435, 2005.

[6] E. Takimoto, H. C. Champion, M. Li et al., "Oxidant stress from nitric oxide synthase-3 uncoupling stimulates cardiac pathologic remodeling from chronic pressure load," Journal of Clinical Investigation, vol. 115, no. 5, pp. 1221-1231, 2005.

[7] F. Cosentino and Z. S. Katusic, "Tetrahydrobiopterin and dysfunction of endothelial nitric oxide synthase in coronary arteries," Circulation, vol. 91, no. 1, pp. 139-144, 1995.

[8] F. Cosentino, D. Hürlimann, C. Delli Gatti et al., "Chronic treatment with tetrahydrobiopterin reverses endothelial dysfunction and oxidative stress in hypercholesterolaemia," Heart, vol. 94, no. 4, pp. 487-492, 2008.

[9] T. Heitzer, K. Krohn, S. Albers, and T. Meinertz, "Tetrahydrobiopterin improves endothelium-dependent vasodilation by increasing nitric oxide activity in patients with Type II diabetes mellitus," Diabetologia, vol. 43, no. 11, pp. 1435-1438, 2000.

[10] S. Setoguchi, M. Mohri, H. Shimokawa, and A. Takeshita, "Tetrahydrobiopterin improves endothelial dysfunction in coronary microcirculation in patients without epicardial coronary artery disease," Journal of the American College of Cardiology, vol. 38, no. 2, pp. 493-498, 2001.

[11] W. Maier, F. Cosentino, R. B. Lütolf et al., "Tetrahydrobiopterin improves endothelial function in patients with coronary artery disease," Journal of Cardiovascular Pharmacology, vol. 35, no. 2, pp. 173-178, 2000.

[12] M. Ozaki, S. Kawashima, T. Yamashita et al., "Overexpression of endothelial nitric oxide synthase accelerates atherosclerotic lesion formation in apoE-deficient mice," Journal of Clinical Investigation, vol. 110, no. 3, pp. 331-340, 2002.

[13] Y. Hattori, S. Hattori, X. Wang, H. Satoh, N. Nakanishi, and K. Kasai, "Oral administration of tetrahydrobiopterin slows the progression of atherosclerosis in apolipoprotein E-knockout mice," Arteriosclerosis, Thrombosis and Vascular Biology, vol. 27, no. 4, pp. 865-870, 2007.

[14] N. J. Alp, S. Mussa, J. Khoo et al., "Tetrahydrobiopterindependent preservation of nitric oxide-mediated endothelial function in diabetes by targeted transgenic GTPcyclohydrolase I overexpression," Journal of Clinical Investigation, vol. 112, no. 5, pp. 725-735, 2003.

[15] J. S. Zheng, X. Q. Yang, K. J. Lookingland et al., "Gene transfer of human guanosine 5 '-triphosphate cyclohydrolase I restores vascular tetrahydrobiopterin level and endothelial function in low renin hypertension," Circulation, vol. 108, no. 10, pp. 1238-1245, 2003. 
[16] S. Wang, J. Xu, P. Song et al., "Acute inhibition of guanosine triphosphate cyclohydrolase 1 uncouples endothelial nitric oxide synthase and elevates blood pressure," Hypertension, vol. 52, no. 3, pp. 484-490, 2008.

[17] K. Goya, S. Sumitani, X. Xu et al., "Peroxisome proliferatoractivated receptor $\alpha$ agonists increase nitric oxide synthase expression in vascular endothelial cells," Arteriosclerosis, Thrombosis and Vascular Biology, vol. 24, no. 4, pp. 658-663, 2004.

[18] Y. Wang, Y. Wang, Q. Yang et al., "Effects of bezafibrate on the expression of endothelial nitric oxide synthase gene and its mechanisms in cultured bovine endothelial cells," Atherosclerosis, vol. 187, no. 2, pp. 265-273, 2006.

[19] K. E. Ryan, D. R. McCance, L. Powell, R. McMahon, and E. R. Trimble, "Fenofibrate and pioglitazone improve endothelial function and reduce arterial stiffness in obese glucose tolerant men," Atherosclerosis, vol. 194, no. 2, pp. e123-e130, 2007.

[20] L. He, H. Zeng, F. Li et al., "Homocysteine impairs coronary artery endothelial function by inhibiting tetrahydrobiopterin in patients with hyperhomocysteinemia," American Journal of Physiology, vol. 299, no. 6, pp. E1061-E1065, 2010.

[21] L. Hel, S. Liu, J. M. Mao et al., "Plasma levels of tetrahydrobiopterin and endothelial function were improved by fenofibrate treatment in patients with hypertriglyceridemia," Circulation, vol. 120, p. S1073, 2009.

[22] F. Lantoine, L. Iouzalen, M. A. Devynck, E. Millanvoye-Van Brussel, and M. David-Dufilho, "Nitric oxide production in human endothelial cells stimulated by histamine requires Ca2+ influx," Biochemical Journal, vol. 330, no. 2, pp. 695-699, 1998.

[23] S. J. Liao, L. Lin, J. S. Zeng, R. X. Huang, K. M. Channon, and A. F. Chen, "Endothelium-targeted transgenic GTPcyclohydrolase I overexpression inhibits neointima formation in mouse carotid artery," Clinical and Experimental Pharmacology and Physiology, vol. 34, no. 12, pp. 1260-1266, 2007.

[24] X. Zeng, J. Dai, D. G. Remick, and X. Wang, "Homocysteine mediated expression and secretion of monocyte chemoattractant protein-1 and interleukin- 8 in human monocytes," Circulation Research, vol. 93, no. 4, pp. 311-320, 2003.

[25] S. Daff, "NO synthase: structures and mechanisms," Nitric Oxide, vol. 23, no. 1, pp. 1-11, 2010.

[26] Y. Hattori, N. Nakanishi, K. Akimoto, M. Yoshida, and K. Kasai, "HMG-CoA reductase inhibitor increases GTP cyclohydrolase I mRNA and tetrahydrobiopterin in vascular endothelial cells," Arteriosclerosis, Thrombosis and Vascular Biology, vol. 23, no. 2, pp. 176-182, 2003.

[27] K. M. Channon, "Tetrahydrobiopterin: regulator of endothelial nitric oxide synthase in vascular disease," Trends in Cardiovascular Medicine, vol. 14, no. 8, pp. 323-327, 2004.

[28] S. Wang, J. Xu, P. Song, B. Viollet, and M. H. Zou, "In vivo activation of AMP-activated protein kinase attenuates diabetes-enhanced degradation of GTP cyclohydrolase I," Diabetes, vol. 58, no. 8, pp. 1893-1901, 2009. 


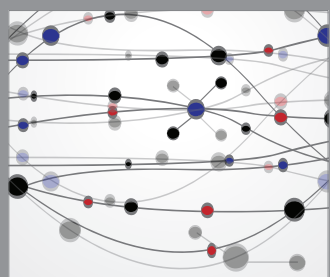

The Scientific World Journal
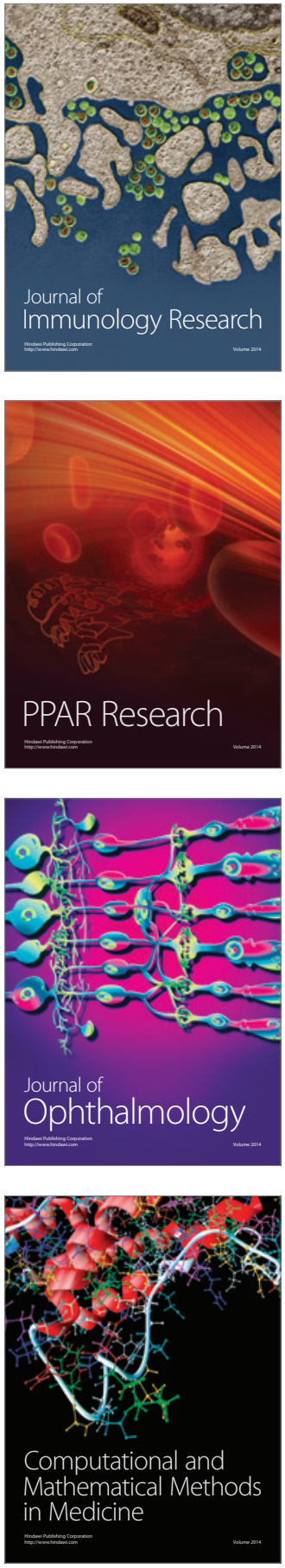

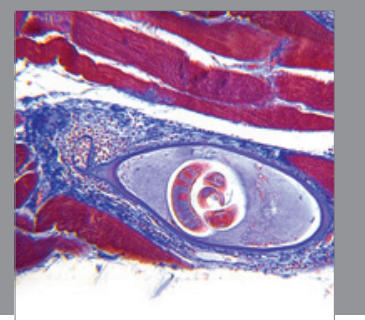

Gastroenterology

Research and Practice
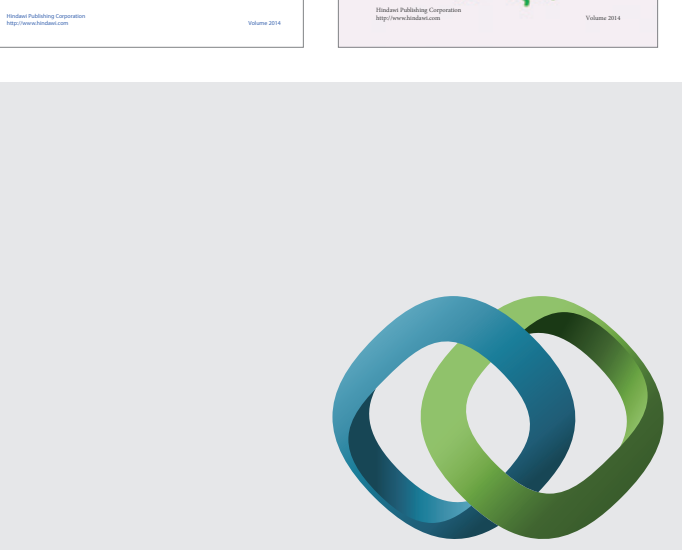

\section{Hindawi}

Submit your manuscripts at

http://www.hindawi.com
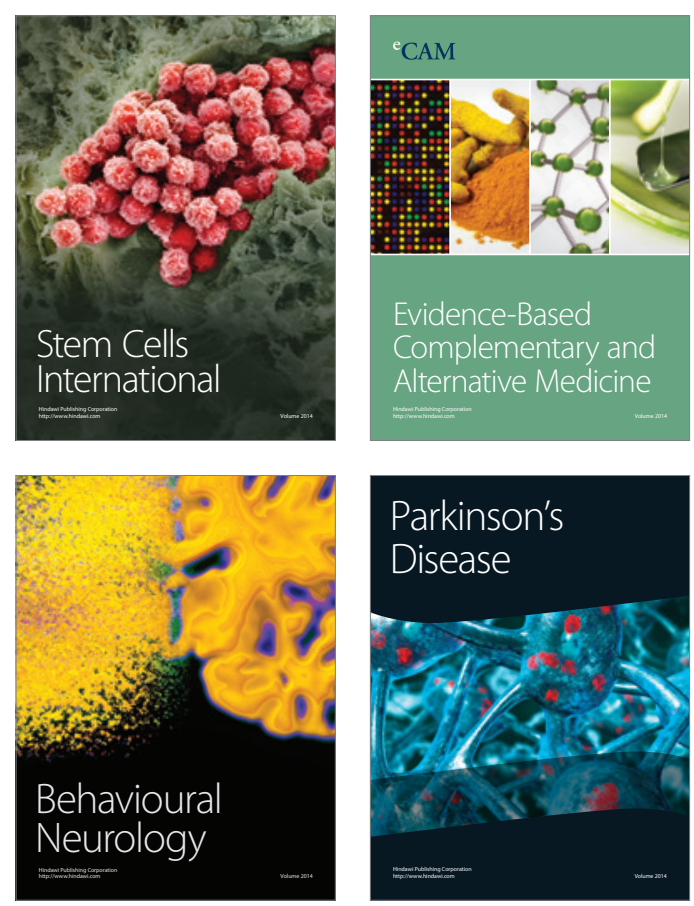

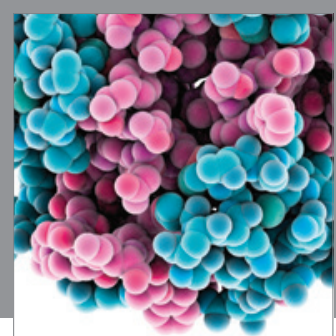

Journal of
Diabetes Research

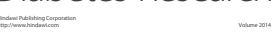

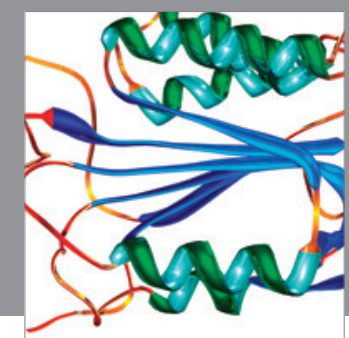

Disease Markers
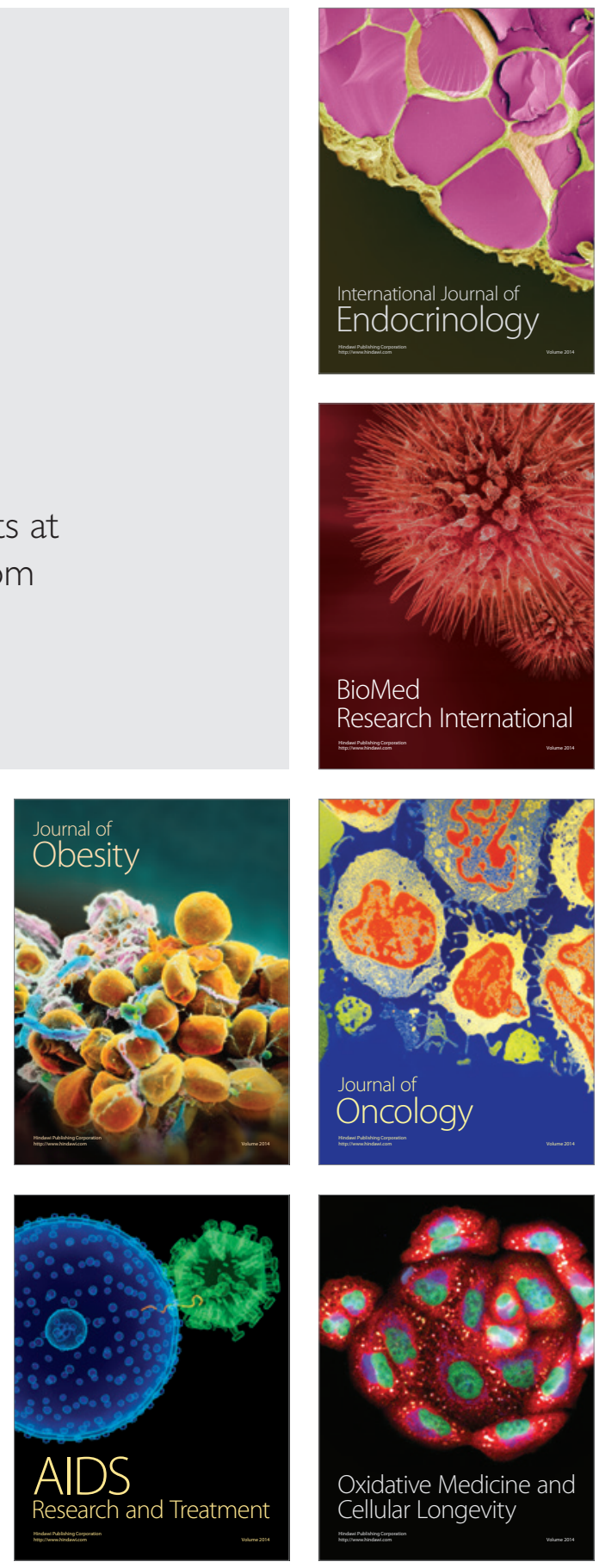